\title{
Nucleocytoplasmic Transport: A Paradigm for Molecular Logistics in Artificial Systems
}

\author{
Suncica Vujica ${ }^{a}$, Christina Zelmer ${ }^{\text {ab }}$, Radhakrishnan Panatalaa, and Roderick Y. H. Limª
}

\begin{abstract}
Artificial organelles, molecular factories and nanoreactors are membrane-bound systems envisaged to exhibit cell-like functionality. These constitute liposomes, polymersomes or hybrid lipo-polymersomes that display different membrane-spanning channels and/or enclose molecular modules. To achieve more complex functionality, an artificial organelle should ideally sustain a continuous influx of essential macromolecular modules (i.e. cargoes) and metabolites against an outflow of reaction products. This would benefit from the incorporation of selective nanopores as well as specific trafficking factors that facilitate cargo selectivity, translocation efficiency, and directionality. Towards this goal, we describe how proteinaceous cargoes are transported between the nucleus and cytoplasm by nuclear pore complexes and the biological trafficking machinery in living cells (i.e. nucleocytoplasmic transport). On this basis, we discuss how biomimetic control may be implemented to selectively import, compartmentalize and accumulate diverse macromolecular modules against concentration gradients in artificial organelles.
\end{abstract}

Keywords: Artificial nuclei · Biomimetics - Macromolecular trafficking · Nuclear pore complex ·

Proteopolymersomes

\section{Introduction}

Living cells are the machines of life. ${ }^{[1]}$ The sophistication of eukaryotic cells is underscored by the prevalence of organelles that encapsulate different contents within specialized membrane-bound microenvironments. ${ }^{[2]}$ This separation from the bulk intracellular space facilitates the co-existence of diverse biochemical reactions that culminate into synergistic cellular functions. To this end, specific proteins are sorted and delivered to exact spatial locations within the complex cellular milieu (i.e. protein targeting ${ }^{[3]}$ in an unprecedented manner. To illustrate this, gene expression is mediated by the spatial separation of transcription and translation that occurs in the nucleus and cytoplasm, respectively. This requires that essential cargoes such as transcription factors are selectively transported across a double bilayer membrane known as the nuclear envelope (NE) that encapsulates the nucleus. ${ }^{[4]}$ This form of intracellular communication is known as nucleocytoplasmic transport (NT), and is mediated by large perforations in the NE known as nuclear pore complexes (NPC). ${ }^{5]}$

\footnotetext{
${ }^{*}$ Correspondence: Prof. Dr. R. Y. H. Lim ${ }^{a}$ E-mail: roderick.lim@unibas.ch

aBiozentrum and the Swiss Nanoscience Institute University of Basel, Klingelbergstrasse 70 $\mathrm{CH}-4056$ Basel

${ }^{\mathrm{b} C h e m i s t r y ~ D e p a r t m e n t, ~ U n i v e r s i t y ~ o f ~ B a s e l ~}$
}

From an abiological perspective, artificial organelles, molecular factories and nanoreactors are membrane-bound molecular systems that are envisaged to replicate or harness the efficacy and complexity of cellular function.[6] This constitutes a shift from a 'one-flask one-reaction' paradigm to a system where cross-regulated reactions take place under spatiotemporal control.[7] However, this introduces basic logistical challenges that remain unaddressed. As a case in point, how are specific proteins and enzymes (henceforth termed 'molecular modules') recruited into an artificial organelle to drive such cross-reactions? In this article, we introduce Nature's solution to the problem of molecular logistics in the context of nucleocytoplasmic transport regulation. Accordingly, we propose that an analogous system may be harnessed to regulate molecular logistics in artificial organelles and other bio-synthetic systems.

\section{Nanoreactors, Artificial Organelles, and Molecular Factories}

Molecular compartments can be constructed from lipid membranes that form liposomes ${ }^{[8]}$ or from amphiphilic polymers that form polymeric membranes and vesicles known as polymersomes. ${ }^{[9]}$ Based on their enhanced mechanical and chemical stability ${ }^{[10]}$ however, polymer-based membranes are increasingly being favored for biomimetic systems ${ }^{[11]}$ and remain the focus of this article. We kindly refer the reader to the review article by Scalise et $a l$. for an in-depth discussion on proteoliposomes. ${ }^{[12]}$

Since their discovery, the encapsulation efficacy of polymersomes has been shown to protect sugars, enzymes or proteins against proteolytic attack. ${ }^{[13]}$ Subsequently, several transmembrane proteins and channels have been successfully inserted into the polymeric membrane to render the polymersome permeable to ions and small molecules, i.e. proteopolymersomes. Importantly, this allows for a product and/or substrate to be exchanged with the outside environment so as to support in situ reactions within the encapsulated entities. ${ }^{[14]}$ For instance, the insertion of gramicidin and ionomycin into the polymersome membrane facilitates the transport of monovalent cations $\left(\mathrm{H}^{+}, \mathrm{Na}^{+} \text {and } \mathrm{K}^{+}\right)^{[15]}$ and divalent cations $\left(\mathrm{Ca}^{2+}\right),{ }^{16]}$ respectively. Moreover, electrical and chemical gradients have been generated by irradiating and activating polymersomes harboring lightdriven proton pumps such as bacteriorhodopsin ${ }^{[17]}$ and proteorhodopsin.[18] The reconstitution of aquaporin-0 (AQP0) further yielded intrinsically osmoregulatory polymersomes that were able to transport water molecules across their polymeric membranes. ${ }^{[19]}$

To transport larger molecules, $\alpha$-hemolysin that bears an inner channel diameter of $\sim 2.6 \mathrm{~nm}$ has enabled the exchange of ions and vital molecules viz., $\mathrm{ATP}^{[20]}$ while molecules of up to $600 \mathrm{Da}$ 
could traverse the $\sim 3.3 \mathrm{~nm}$-diameter outer membrane protein $\mathrm{F}(\mathrm{OmpF})$ to diffuse into the polymersomes. ${ }^{[13 \mathrm{~b}]}$ To further biocatalyze reactions, ${ }^{[21]} \mathrm{OmpF}$ was chemically modified with a molecular 'cap' that acted as a $\mathrm{pH}$ responsive gate and could be opened by lowering the $\mathrm{pH}$. In this manner, a modified OmpF-bearing polymersome could serve as a nanoreactor by converting the influx of chromogenic substrate 3,3',5,5'-tetramethylbenzidine (TMB) using a pre-encapsulated model enzyme horseradish peroxidase (HRP) within its lumen. Further, entrapping antioxidant enzymes within a polymeric membrane that was leaky to reactive oxygen species (ROS) could effectively accelerate ROS detoxification within cells under oxidative stress. ${ }^{[22]}$ As a step towards multicompartmentalization, two different nanoreactors bearing two different enzymes could be encapsulated into a larger polymersome ${ }^{[23]}$ and used to control a cascade reaction across the compartmental boundaries. ${ }^{[24]}$ We refer the interested reader to Table 1 for a summary of several transmembrane channels that have been engineered into polymersomes. For a detailed overview of existing protein reconstitution protocols into polymersomes we refer the reader to the review by Garni et al. in this special issue.[25]

In spite of the abovementioned breakthroughs, we note that transport into proteopolymersomes is largely restricted to ions and small molecules based on size exclusion as defined by the inner diameter of the membrane channel. In contrast, an ideal molecular factory would resemble a cell where a number of molecular modules would perform different activities. However, to attain a more cell-like system, it will be necessary to transport diverse substances into and out of a proteopolymersome, depending on the following:

i) How many different types of molecular modules, enzymes and essential proteins are needed to construct a functional 'molecular factory'?

ii) What are the optimal concentrations needed for such molecular modules to function?

iii) How large are the molecular modules under consideration, and how should they be encapsulated or imported?

iv) How will the transport of essential reactants, ligands and metabolites be regulated?

v) How will reaction byproducts be removed from the proteopolymersome interior to the external environment?

vi) What can be used as an energy source to sustain the longevity and turnover of such reactions?

Hence, this underscores the need for a more advanced form of molecular logistics that goes beyond passive diffusion and size exclusion. Such a molecular logistic system should facilitate the continuous transport of both large and small cargoes, from proteins to metabolites based on biochemical specificity and not size exclusion per $s e$. Moreover, this process should include an energy supply so as to dictate the directionality of transport, to accumulate car-

Table 1. Selection of polymersomes that harbor transmembrane channels and their cargoes.

\section{Transmembrane Channel}

\section{Channel Pore Diameter}

\begin{tabular}{|c|}
\hline Outer \\
\hline 2 \\
\hline
\end{tabular}

Gramicidin

Ionomycin

$\sim 19 \AA$

\begin{tabular}{l|c|c|c|c|}
$\begin{array}{l}\text { Outer } \\
\text { membrane } \\
\text { protein F }\end{array}$ & $\sim 33 \AA$ & $\sim 70 \AA$ & $\sim 39 \mathrm{kDa}$ & $\begin{array}{c}\text { Non-specific } \\
\text { transport } \\
\text { channel }\end{array}$ \\
\hline
\end{tabular}

protein

$(\mathrm{OmpF})$

Proteo-
rhodopsin
(PR)
$\sim 20 \AA$

$\sim 87 \AA$

Bacterio-

rhodopsin

(BR)

Aquaporin-0

$\sim 1.5 \AA$

(AQP0)

$\alpha$-Hemolysin $\sim 100 \AA$ $\sim 0.8 \mathrm{kDa}$

Calciumtransporter,

Antibiotic

$\sim 29 \mathrm{kDa}$

Molecular
Weight
$\sim 1.9 \mathrm{kDa}$

Biological

Proton pump,

Antibiotic

channel

proton pump

$\sim 43 \AA$

$\sim 27 \mathrm{kDa}$

Light-driven
proton pump

proton pump

8 А

$\sim 28 \mathrm{kDa}$

Water channel

Toxin

secretion

systems
Small

molecules

$<600 \mathrm{Da}$

e.g.

ampicillin,

TMB

[28]

[29]

\section{Transported \\ ubstrate/ \\ Monovalent ons}

$\mathrm{H}^{+}$

$\mathrm{H}^{+}$

$\mathrm{H}_{2} \mathrm{O}$

[30]

Ions, small $\sim 32 \mathrm{kDa}$
Reconstituted Membrane

Compound Ref.

PMOXAPDMS-

PMOXA $^{\mathrm{a}}$

PMOXAPDMSPMOXA $^{\mathrm{a}}$

PMOXA-

PDMS-

PMOXA $^{a}$

PBD-

P4MVP

PEtOz-

PDMS-

$\mathrm{PEtOz}^{\mathrm{c}}$

PMOXA-

PDMS-

PMOXA $^{\mathrm{a}}$

PBD-PEO

molecules

e.g. toxins,

single DNA 
goes within the proteopolymersome (i.e. against concentration gradients), as well as to ensure an efficient turnover of reaction byproducts. Quite remarkably, these key features are known to regulate NT in eukaryotic cells. In the following section, we give insight as to how living cells selectively import, compartmentalize and accumulate diverse macromolecular modules against concentration gradients.

\section{Nucleocytoplasmic Transport and the Nuclear Pore Complex}

In eukaryotic cells, the nucleus communicates exclusively with the cytoplasm through proteinaceous pores termed nuclear pore complexes (NPCs). ${ }^{[31]}$ NPCs mediate the nucleocytoplasmic trafficking of substances ranging from the import of transcription factors to mRNA export. ${ }^{[32]}$ It is estimated that approximately one thousand translocation events proceed per NPC per second ${ }^{[33]}$ at a rate of $\sim 5 \mathrm{~ms}$ per cargo. ${ }^{[34]}$ NPCs are constructed from approximately 30 proteins known as nucleoporins (Nups) that are present in multiples of eight ${ }^{[35]}$ and amount to an overall mass of $\sim 60 \mathrm{MDa}^{[36]}$ and $\sim 120 \mathrm{MDa}^{[37]}$ in yeast and in metazoans, respectively. Nups are hierarchically categorized into three architectural subgroups:[38] i) membrane Nups that anchor the NPC to the NE; ii) structural scaffold Nups; and, iii) a family of intrinsically disordered Phe-Gly (FG) Nups.

AlthoughNPCs span species-dependent diameters of between 50 to $100 \mathrm{~nm}^{[39]}$ only small molecules below $\sim 40 \mathrm{kDa}$ (e.g. water and ions) can passively diffuse through the pore whereas large non-specific macromolecules are generally withheld. ${ }^{[40]}$ Rapid and exclusive access through the NPC is permissible only to transport receptors ${ }^{[41]}$ (i.e. karyopherins or Kaps) that exceed the passive limit, such as the classical $97 \mathrm{kDa}$ import receptor karyopherin $\beta 1$ (Kap $\beta 1$ or importin $\beta^{[42]}$ or Kap95 in yeast). Hence, NPCs restrict or promote cargo translocation via biochemical selectivity and not size exclusion per se.

Altogether $\sim 200$ FG Nup molecules ${ }^{[35]}$ tether to the inner walls of the NPC and manifest the NPC barrier that facilitates selective transport control. Being intrinsically disordered and lacking in secondary structure, the 'polymer-like' FG Nups are thought to resemble a 'supramolecular hydrogel meshwork' [43] and/or a 'molecular brush' barrier, ${ }^{[44]}$ or combinations thereof. ${ }^{[45]}$ To bypass the FG Nup barrier, Kaps and other transport factors, ${ }^{[42,46]}$ harbor binding sites that interact multivalently with the FG-repeat motifs (i.e. GLFG, FxFG, and FG) that decorate the FG Nups. This binding facilitates Kap transport by causing dynamic conformational chang- es in the FG Nup barrier to open up the pore. ${ }^{[47]}$ On this basis, Kaps orchestrate nucleocytoplasmic transport by authenticating and shuttling specific cargoes destined for the nucleus through the NPCs.

Kaps identify specific cargoes via short peptide sequences known as nuclear localization signals, or nuclear export signals (NLSs or NESs). ${ }^{[4]}$ Thus, non-specific macromolecules, which lack NLS or NES are rejected by the barrier.[41] On the other hand, by recruiting Kaps, very large physiological as well as artificial cargoes with sizes that span the inner diameter of the pore are able to translocate the NPC. These include gold particles decorated with Kap-cargo complexes (39 nm-diameter overall), ${ }^{[49]}$ Kap-decorated quantum dot cargoes (30 to $40 \mathrm{~nm}$-diameter overall), ${ }^{[50]}$ Hepatitis B virus capsids (34 nm-diameter), ${ }^{[51]}$ and messenger ribonucleoproteins (mRNPs; e.g. Balbani Ring particles), which appear to elongate to $25 \mathrm{~nm}$-diameter during passage through the central channel.[52] Indeed, viruses are known to hijack the Kap-FG pathway in order to infiltrate the NPC and the nucleus. ${ }^{[53]}$
During import (Fig. 1), Kap-cargo translocation is itself an energy-independent diffusional process. ${ }^{[54]}$ However, an energy supply is required to establish cargo directionality and the termination of transport. ${ }^{[55]}$ Moreover, a continuous energy cycle is required to sustain nucleocytoplasmic trafficking. This is regulated by the small GTPase Ran, which has GTP- and GDPbound forms localized to the nucleus and cytoplasm, respectively. ${ }^{[56]}$ RanGTP binding triggers the release of import cargoes from Kap $\beta 1$ in the nucleus while in the cytoplasm RanGAP (Ran GTPase-activating protein) hydrolyzes RanGTP to RanGDP, which releases Kap $\beta 1$ for another cargo import cycle. ${ }^{[57]}$ RanGDP is then recycled to the nucleus by its specific carrier, NTF2 (i.e. nuclear transport factor 2). ${ }^{[58]}$ In the absence of Kap $\beta 1$, even NLS-cargoes that are smaller than entire Kap-cargo complexes are withheld. Accordingly, NLScargoes are retained in the nucleus upon dissociation from Kap $\beta 1$. The Ran loop is finally closed by RCC1/RanGEF (guanine nucleotide exchange factor), which catalyzes the recharging of RanGDP to

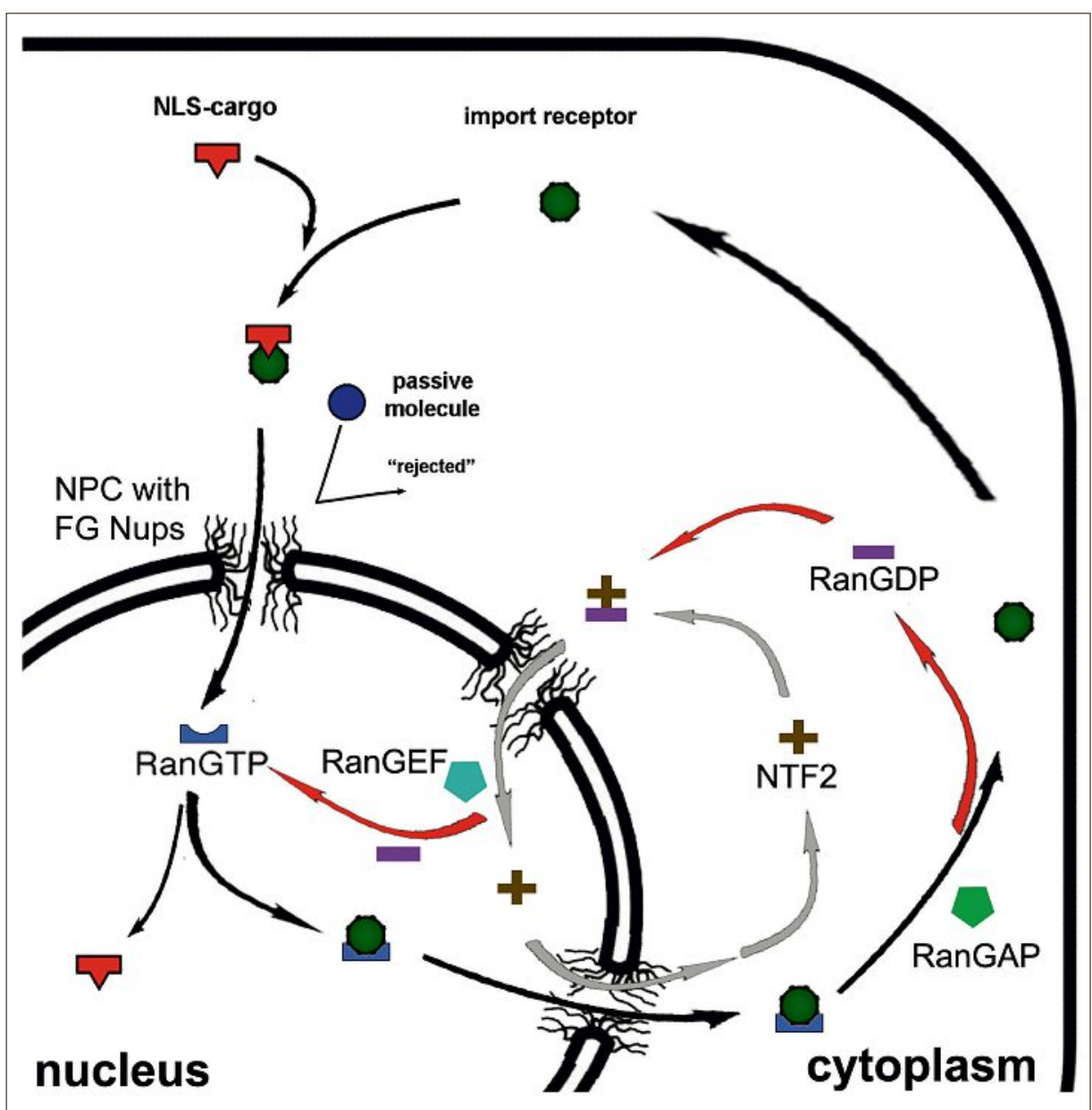

Fig. 1. Mechanism of nuclear import. Import receptors (Kap $\beta 1$ ) identify and shuttle NLS-cargo from the cytoplasm into the nucleus. The receptor-cargo complex is disassembled in the nucleus by RanGTP, which returns with the import receptor to the cytosol. RanGAP triggers the hydrolysis of RanGTP to RanGDP in the cytosol, which frees the receptor for another round of import. RanGDP is imported into the nucleus by NTF2, where it is recharged into RanGTP by RanGEF. In the absence of Kaps, non-specific cargoes are rejected by the NPC. 
RanGTP. ${ }^{57]}$ Hence, RanGAP and RCC1 enable the cell to maintain high levels of RanGTP inside the nucleus, and low levels of it in the cytoplasm. This drives the transport and accumulation of cargoes against their concentration gradients within the nucleus (Fig. 2)

\section{Towards 'Artificial Nuclei'}

The mechanism of nucleocytoplasmic transport highlights several hierarchical principles ${ }^{[59]}$ that surpass basic encapsulation/size exclusion and may be applied in artificial systems. In fact, solid-state nanopores and polymeric membranes that incorporate FG Nups (artificial NPCs) have been shown to exert NPC-like properties. ${ }^{[60]}$ FG Nup-functionalized surfaces have also been shown to reconstitute selective transport in two dimensions.[61] Accordingly, this presents a molecular logistics paradigm for regulating transport and communication between artificial organelles, i.e. so-called 'artificial nuclei',
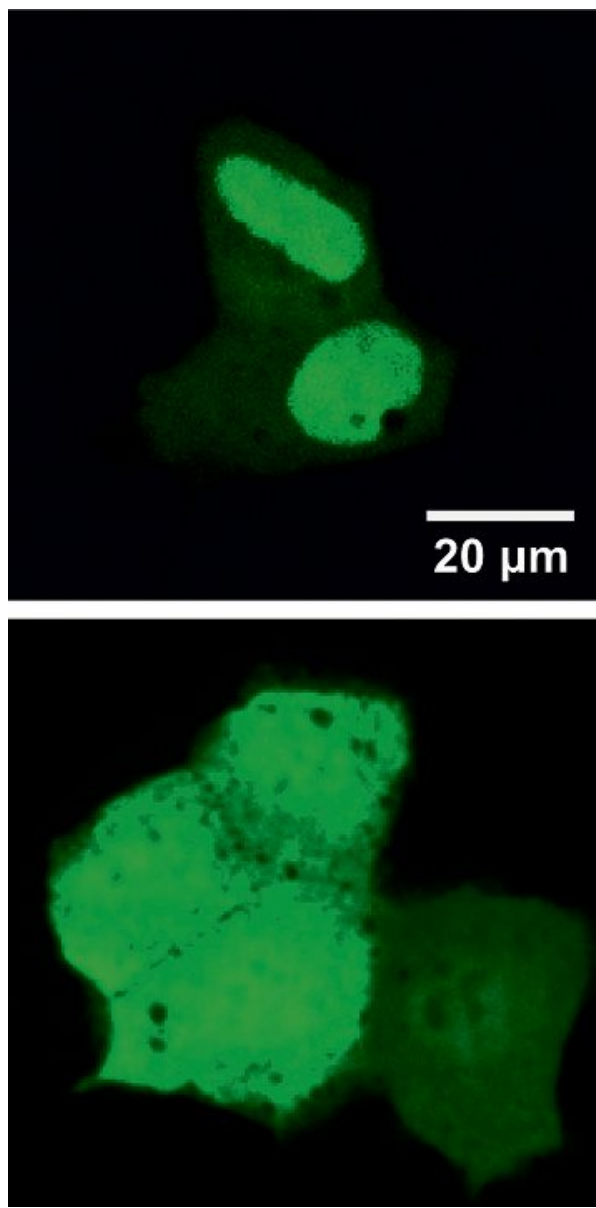

Fig. 2. NLS-modified green fluorescent proteins (GFPs) are selectively trafficked by transport receptors across NPCs and accumulate against concentration gradients within the nuclei of living cells (top). In contrast, GFP molecules lacking the NLS are homogenously distributed throughout entire cells (bottom). Scale bar $=20 \mu \mathrm{m}$ which can be broken down into the following constituent parts:

i) NPC-like selective nanopores that are large enough (on the order of $10 \mathrm{~nm}$ ) to accommodate a diverse range of molecular entrants of varying sizes. To prevent unwanted infiltration, each nanopore should be assembled with a stimuli-responsive 'barrier' that regulates the permeability and porosity of the 'artificial nuclei'. A priori the barrier repels non-specific entities but facilitates the translocation of molecular 'keys' (see below) based on (bio) chemical recognition. This further imparts a property of anti-fouling that prevents non-specific macromolecules from clogging the pores within a complex milieu.[62] Thus, biosynthetic alternatives to the FG Nups might include chemically-derivatized biorecognition polymers. ${ }^{[63]}$

ii) Kap-like molecular 'keys' that gain selective and exclusive access through the nanopore by exerting specific biochemical interactions with the stimuli-responsive barrier. Additionally, such 'keys' have to recognize and bind molecular modules, i.e. the main cargoes of interest (see below). Possible candidates include bispecific antibodies $^{[64]}$ (or other antibody variants). ${ }^{[65]}$

iii) A suite of cargoes (e.g. molecular modules, enzymes, other essential proteins) that do not themselves interact with the stimuli-responsive barrier but instead bear signal-specific tags (e.g. NLS) that the molecular keys recognize and bind to. In this manner, cargoes traverse the nanopore by 'hitchhiking' along with the molecular key.

iv) Employing a 'host' such as RanGTP inside the 'artificial nuclei' that facilitates cargo release from the key upon entering the luminal space. After encapsulation, an outflow of cargo is minimized for the same reason that a key was required to enter the 'artificial nucleus' in the first place.

v) An energy source that provides feedback and recycles the keys and replenishes the 'host'. This ensures transport directionality, continuity and accumulation against concentration gradients.

Based on the above criteria, we postulate that a mechanism of molecular logistics can be implemented in bio-synthetic systems. As a basic proof-of-principle of points $\mathrm{i}$ to iii, we previously used PEGantibodies as bio-hybrid molecular keys to sift secondary antibodies out of serum to non-fouling PEGylated targets. ${ }^{[66]}$ Yet, perhaps the most interesting and challenging feature of constructing an 'artificial nucleus' involves the implementation of an energy cycle. In living cells, it is essential to maintain RanGTP/RanGDP asymmetry in order to accumulate cargoes against concentration gradients in nuclei. ${ }^{[67]}$ Presently however, an in vitro energy source has yet to be identified.

\section{Outlook}

The essence of our hypothesis lies in being able to translate a biological transport mechanism into an abiological molecular logistics system. Such a system would have the capability to sort and encapsulate specific molecular modules from a complex environment into artificial organelles leading to the construction of a molecular factory. Importantly, we note that such a molecular logistics system takes a 'one size fits all' approach requiring only that the cargoes-of-interest bear signal specific tags that are recognized by the molecular keys. In this manner, the system can be easily adapted for constructing different molecular factories by trafficking molecular modules of pre-determined functionality. Further refinements to such a system could include exploiting reaction by-products as a feedback parameter (e.g. to increase transport levels) or as a means of communication between different molecular factories. Nevertheless, an immediate challenge will be to identify suitable molecular building blocks from which such a molecular logistics system can be assembled. Moreover, it will be essential to study the reactions that take place under off-equilibrium thermodynamic conditions within a crowded and confined aqueous environment.

\section{Acknowledgements}

We are grateful to K.D. Schleicher for the fluorescence images. Our work is supported by the Biozentrum, the Swiss Nanoscience Institute, and the Swiss National Science Foundation as part of the NCCR in Molecular Systems Engineering.

Received: April 16, 2016

[1] D. S. Goodsell, 'The machinery of life', Springer Science \& Business Media, 2009.

[2] G. M. Cooper, R. E. Hausman, 'The cell', Sinauer Associates Sunderland, 2000.

[3] G. Blobel, ChemBiochem 2000, 1, 87.

[4] S. R. Wente, M. P. Rout, Cold Spring Harb. Perspect. Biol. 2010, 2, a000562.

[5] M. Beck, F. Forster, M. Ecke, J. M. Plitzko, F. Melchior, G. Gerisch, W. Baumeister, O. Medalia, Science 2004, 306, 1387.

[6] P. R. Leduc, M. S. Wong, P. M. Ferreira, R. E. Groff, K. Haslinger, M. P. Koonce, W. Y. Lee, J. C. Love, J. A. McCammon, N. A. MonteiroRiviere, V. M. Rotello, G. W. Rubloff, R. Westervelt, M. Yoda, Nat Nanotechnol. 2007, 2,3.

[7] K. E. Drexler, Trends Biotechnol. 1999, 17, 5.

[8] a) D. M. Engelman, Nature 2005, 438, 578; b) G. Van Meer, D. R. Voelker, G. W. Feigenson, Nature Rev. Mol. Cell Biol. 2008, 9, 112.

[9] a) H. A. Klok, S. Lecommandoux, Adv. Mater. 2001, 13, 1217; b) J. Kowal, X. Zhang, I. A. Dinu, C. G. Palivan, W. Meier, ACS Macro Lett. 2013, 3, 59

[10] D. E. Discher, A. Eisenberg, Science 2002, 297, 967.

[11] C. LoPresti, H. Lomas, M. Massignani, T. Smart, G. Battaglia, J. Mater. Chem. 2009, 19, 3576. 
[12] M. Scalise, L. Pochini, N. Giangregorio, A. Tonazzi, C. Indiveri, Pharmaceutics 2013, 5, 472.

[13] a) W. Meier, C. Nardin, M. Winterhalter, Angew. Chem. Int. Ed. 2000, 39, 4599; b) C. Nardin, J. Widmer, M. Winterhalter, W. Meier, Eur. Phys. J. E 2001, 4, 403.

[14] V. Balasubramanian, O. Onaca, R. Enea, D. W. Hughes, C. G. Palivan, Exp. Opin. Drug Deliv. 2010, 7, 63.

[15] M. Lomora, M. Garni, F. Itel, P. Tanner, M. Spulber, C. G. Palivan, Biomater. 2015, 53, 406

[16] M. Lomora, F. Itel, I. A. Dinu, C. G. Palivan, PhysChemChemPhys 2015, 17, 15538.

[17] H.-J. Choi, C. D. Montemagno, Nanotechnol. IEEE Trans. 2007, 6, 171.

[18] D. Hua, L. Kuang, H. Liang, J. Am. Chem. Soc. 2011, 133, 2354.

[19] R. Stoenescu, A. Graff, W. Meier, Macromol. Biosci. 2004, 4, 930.

[20] M. Nallani, M. Andreasson-Ochsner, C.-W. D. Tan, E.-K. Sinner, Y. Wisantoso, S. GeifmanShochat, W. Hunziker, Biointerphases 2011, 6, 153.

[21] T. Einfalt, R. Goers, I. A. Dinu, A. Najer, M. Spulber, O. Onaca-Fischer, C. G. Palivan, Nano Lett. 2015, 15, 7596.

[22] P. Tanner, V. Balasubramanian, C. G. Palivan, Nano Lett. 2013, 13, 2875.

[23] A. L. Klyszejko, S. Shastri, S. A. Mari, H. Grubmüller, D. J. Muller, C. Glaubitz, J. Mol. Biol. 2008, 376, 35.

[24] P. Tanner, O. Onaca, V. Balasubramanian, W. Meier, C. G. Palivan, Chem. Eur. J. 2011, 17, 4552.

[25] M. Garni, T. Einfalt, M. Lomora, A. Car, W. Meier, C. G. Palivan, Chimia 2016, 70, 424.

[26] D. A. Kelkar, A. Chattopadhyay, Biochim. Biophys. Acta Biomembranes 2007, 1768, 2011.

[27] V. Chaptal, A. Kilburg, D. Flot, B. Wiseman, N. Aghajari, J.-M. Jault, P. Falson, Biochim Biophys. Acta Biomembranes 2016, 1858, 326.

[28] R. Peters, R. J. Cherry, Proc. Natl. Acad. Sci. USA 1982, 79, 4317

[29] K. Murata, K. Mitsuoka, T. Hirai, T. Walz, P. Agre, J. B. Heymann, A. Engel, Y. Fujiyoshi, Nature 2000, 407, 599.

[30] L. Song, M. R. Hobaugh, C. Shustak, S. Cheley, H. Bayley, J. E. Gouaux, Science 1996, 274, 1859.

[31] G. Kabachinski, T. U. Schwartz, J. Cell Sci. 2015, 128, 423.

[32] a) B. Fahrenkrog, U. Aebi, Nat. Rev. Mol. Cell Biol. 2003, 4, 757; b) E. J. Tran, S. R. Wente, Cell 2006, 125, 1041.
[33] K. Ribbeck, D. Gorlich, EMBO J. 2001, 20, 1320

[34] T. Dange, D. Grunwald, A. Grunwald, R. Peters, U. Kubitscheck, J. Cell Biol. 2008, 183, 77.

[35] J. M. Cronshaw, A. N. Krutchinsky, W. Zhang, B. T. Chait, M. J. Matunis, J. Cell Biol. 2002, $158,915$.

[36] M. P. Rout, G. Blobel, J. Cell Biol. 1993, 123, 771.

[37] R. Reichelt, A. Holzenburg, E. L. Buhle, Jr., M. Jarnik, A. Engel, U. Aebi, J. Cell Biol. 1990, 110,883

[38] D. Devos, S. Dokudovskaya, R. Williams, F. Alber, N. Eswar, B. T. Chait, M. P. Rout, A. Sali, Proc. Natl. Acad. Sci. USA 2006, 103, 2172.

[39] a) N. Elad, T. Maimon, D. Frenkiel-Krispin, R. Y. Lim, O. Medalia, Curr. Opin. Struct. Biol. 2009, 19, 226; b) D. Frenkiel-Krispin, B. Maco, U. Aebi, O. Medalia, J. Mol. Biol. 2010, 395, 578; c) D. Stoffler, B. Feja, B. Fahrenkrog, J. Walz, D. Typke, U. Aebi, J. Mol. Biol. 2003, 328, 119.

[40] P. Popken, A. Ghavami, P. R. Onck, B. Poolman, L. M. Veenhoff, Mol. Biol. Cell 2015, 26, 1386.

[41] Y. M. Chook, K. E. Suel, Biochim. Biophys. Acta 2011, 1813, 1593.

[42] R. Bayliss, T. Littlewood, M. Stewart, Cell 2000, 102, 99.

[43] a) S. Frey, D. Gorlich, Cell 2007, 130, 512; b) B. B. Hulsmann, A. A. Labokha, D. Gorlich, Cell 2012, 150, 738.

[44] R. Y. Lim, N. P. Huang, J. Koser, J. Deng, K. H. Lau, K. Schwarz-Herion, B. Fahrenkrog, U. Aebi, Proc. Natl. Acad. Sci. USA 2006, 103, 9512.

[45] J. Yamada, J. L. Phillips, S. Patel, G. Goldfien, A. Calestagne-Morelli, H. Huang, R. Reza, J. Acheson, V. V. Krishnan, S. Newsam, A. Gopinathan, E. Y. Lau, M. E. Colvin, V. N. Uversky, M. F. Rexach, Mol. Cell Proteomics 2010, 9, 2205.

[46] a) R. Bayliss, S. W. Leung, R. P. Baker, B. B. Quimby, A. H. Corbett, M. Stewart, EMBO J. 2002, 21, 2843; b) R. Bayliss, T. Littlewood, L. A. Strawn, S. R. Wente, M. Stewart, J. Biol. Chem. 2002, 277, 50597; c) R. Bayliss, K. Ribbeck, D. Akin, H. M. Kent, C. M. Feldherr, D. Gorlich, M. Stewart, J. Mol. Biol. 1999, 293, 579 .

[47] a) L. E. Kapinos, R. L. Schoch, R. S. Wagner, K. D. Schleicher, R. Y. Lim, Biophys. J. 2014, 106, 1751; b) R. Y. Lim, B. Fahrenkrog, J. Koser, K. Schwarz-Herion, J. Deng, U. Aebi, Science 2007, 318, 640; c) R. S. Wagner, L. E. Kapinos, N. J. Marshall, M. Stewart, R. Y. Lim, Biophys. J. 2015, 108, 918.
[48] T. Boulikas, J. Cell. Biochem. 1994, 55, 32.

[49] N. Pante, M. Kann, Mol. Biol. Cell 2002, 13, 425.

[50] A. R. Lowe, J. J. Siegel, P. Kalab, M. Siu, K Weis, J. T. Liphardt, Nature 2010, 467, 600.

[51] B. Rabe, A. Vlachou, N. Pante, A. Helenius, M. Kann, Proc. Natl. Acad. Sci. USA 2003, 100, 9849.

[52] H. Mehlin, B. Daneholt, U. Skoglund, Cell 1992, 69, 605.

[53] G. R. Whittaker, M. Kann, A. Helenius, Annu. Rev Cell Dev. Biol. 2000, 16, 627.

[54] R. B. Kopito, M. Elbaum, Proc. Natl. Acad. Sci. USA 2007, 104, 12743.

[55] E. D. Schwoebel, B. Talcott, I. Cushman, M. S Moore, J. Biol.Chem. 1998, 273, 35170.

[56] D. Görlich, I. W. Mattaj, Science 1996, 271 , 1513.

[57] M. Stewart, Nat. Rev. Mol. Cell Biol. 2007, 8, 195.

[58] K. Ribbeck, G. Lipowsky, H. M. Kent, M Stewart, D. Gorlich, EMBO J. 1998, 17, 6587.

[59] L. J. Terry, E. B. Shows, S. R. Wente, Science 2007, 318, 1412

[60] a) T. Jovanovic-Talisman, J. Tetenbaum-Novatt, A. S. McKenney, A. Zilman, R. Peters, M. P. Rout, B. T. Chait, Nature 2009, 457, 1023; b) S. W. Kowalczyk, L. Kapinos, T. R. Blosser, T. Magalhães, P. van Nies, R. Y. Lim, C. Dekker, Nature Nanotechnol. 2011, 6, 433.

[61] K. D. Schleicher, S. L. Dettmer, L. E. Kapinos, S. Pagliara, U. F. Keyser, S. Jeney, R. Y. Lim, Nature Nanotechnol. 2014, 9, 525.

[62] P. Wu, D. G. Castner, D. W. Grainger, J. Biomater. Sci. Polym. Ed. 2008, 19, 725.

[63] R. Barbey, L. Lavanant, D. Paripovic, N Schuwer, C. Sugnaux, S. Tugulu, H. A. Klok, Chem. Rev. 2009, 109, 5437.

[64] A. Pluckthun, P. Pack, Immunotechnol. 1997, 3 83.

[65] A. C. Chan, P. J. Carter, Nat. Rev. Immunol. 2010, 10, 301.

[66] J. T. Hyotyla, J. Deng, R. Y. Lim, ACS Nano 2011, 5, 5180 .

[67] M. V. Nachury, K. Weis, Proc. Natl. Acad. Sci. USA 1999, 96, 9622 . 